\title{
Establishment and Validation of a Nomogram to Predict Hospital-Acquired Infection in Elderly Patients After Cardiac Surgery
}

\author{
Yuchen Gao',*, Chunrong Wang',*, Yuefu Wang', Jun Li', Jianhui Wang', Sudena Wang', Yu Tian', \\ Jia Liu', Xiaolin Diao ${ }^{3}$, Wei Zhao ${ }^{3}$ \\ 'Department of Anesthesiology, State Key Laboratory of Cardiovascular Disease, Fuwai Hospital, National Center of Cardiovascular Diseases, \\ Chinese Academy of Medical Sciences and Peking Union Medical College, Beijing, People's Republic of China; ${ }^{2}$ Department of Anesthesiology and \\ Surgical Intensive Care Unit, Beijing Shijitan Hospital, Capital Medical University, Beijing, People's Republic of China; ${ }^{3}$ Department of Information \\ Center, Skate Key Laboratory of Cardiovascular Disease, Fuwai Hospital, National Center of Cardiovascular Diseases, Chinese Academy of Medical \\ Sciences and Peking Union Medical College, Beijing, People's Republic of China
}

*These authors contributed equally to this work

Correspondence: Yuefu Wang, Department of Anesthesiology and Surgical Intensive Care Unit, Beijing Shijitan Hospital, Capital Medical University, 10 Tieyi Road, Haidian District, Beijing, People's Republic of China, Email wangyuefu3806@bjsjth.cn

Background: Hospital-acquired infection (HAI) after cardiac surgery is a common clinical concern associated with adverse prognosis and mortality. The objective of this study is to determine the prevalence of HAI and its associated risk factors in elderly patients following cardiac surgery and to build a nomogram as a predictive model.

Methods: We developed and internally validated a predictive model from a retrospective cohort of 6405 patients aged $\geq 70$ years, who were admitted to our hospital and underwent cardiac surgery. The primary outcome was HAI. Multivariable logistic regression analysis was used to identify independent factors significantly associated with HAI. The performance of the established nomogram was assessed by calibration, discrimination, and clinical utility. Internal validation was achieved by bootstrap sampling with 1000 repetitions to reduce the overfit bias.

Results: Independent factors derived from the multivariable analysis to predict HAI were smoking, myocardial infarction, cardiopulmonary bypass use, intraoperative erythrocytes transfusion, extended preoperative hospitalization days and prolonged duration of mechanical ventilation postoperatively. The derivation model showed good discrimination, with a C-index of 0.706 [95\% confidence interval 0.671-0.740], and good calibration [Hosmer-Lemeshow test $P=0.139$ ]. Internal validation also maintained optimal discrimination and calibration. The decision curve analysis revealed that the nomogram was clinically useful.

Conclusions: We developed a predictive nomogram for postoperative HAIs based on routinely available data. This predictive tool may enable clinicians to achieve better perioperative management for elderly patients undergoing cardiac surgery but still requires further external validation.

Keywords: prediction, model, older patients, nosocomial infection

\section{Introduction}

Cardiac surgery for elderly individuals is routinely performed in clinical scenarios, and advanced age is no longer a barrier when selecting surgical candidates given that an optimally perioperative scheme can be achieved. ${ }^{1}$ However, elderly individuals are still considered a vulnerable group because they carry a higher proportion of comorbidities than younger individuals and often have severe heart disease. ${ }^{2,3}$

Hospital-acquired infection (HAI), a relatively common complication after cardiac operations, is an important cause of prolonged hospital stays and higher concomitant costs. ${ }^{4-6}$ Cardiac patients are promisingly perceived as a risky subset to encounter postoperative HAI, as they vary greatly from those with noncardiac procedures due in part to the frequent 
utilization of invasive monitoring procedures, including venous and arterial catheters, prolonged mechanical ventilation time and, specifically, exposure to cardiopulmonary bypass (CPB). ${ }^{7,8}$ We preferred elderly patients as our purposed study candidates, which is rooted in population aging, as advanced age is gradually predominating worldwide. It is additionally expected that they have a greater likelihood of developing HAI following cardiac surgery due to age-related pathophysiological mechanisms. This subsequently underlines a major need to identify the causative factors for HAI in elderly individuals and to determine which of these factors can be modified.

A nomogram is a pictorial representation of a scoring model that possesses a simple user interface and shows favorable predictive performance. ${ }^{9}$ By integrating a patient's diverse determinant variables, a nomogram can provide a personalized probability for quantitative predictions of the incidence of an outcome of interest. ${ }^{10}$ However, no predictive model for HAI after cardiac surgery in elderly patients has been described in the previous literature. Therefore, we conducted a retrospective study to establish a nomogram to predict the HAI in elderly patients undergoing cardiac surgery.

\section{Methods}

\section{Study Design and Data Collection}

This is a retrospective study performed at our hospital, approved by the Ethics Committee of Fuwai hospital with waived written consent. We screened adult patients with cardiac lesions who underwent invasive surgical repair from 2012 to 2019. For the study purpose, only elderly individuals older than 70 years old were initially considered. Additionally, patients who had infection before surgery, missing records on postoperative infectious evidence and non-open cardiac procedures were excluded (Figure 1). Patient baseline characteristics, intraoperative information and postoperative outcomes in our database were all extracted from electronic medical records. Although this study was retrospective in nature, all data involved were input prospectively by medical personnel at our institute and then retained.

The routine protocols for patients with regard to general anesthesia and postoperative ICU management are almost standardized at our institute, although subtle variance cannot be denied given attending clinician preferences and individual patient pathology. Prophylactic antibiotics were administered to each patient with $1.5 \mathrm{~g}$ cefuroxime or $600 \mathrm{mg}$ clindamycin before surgery. An additional dose of $0.75 \mathrm{~g}$ cefuroxime was administered for procedures lasting more than 3 hours.

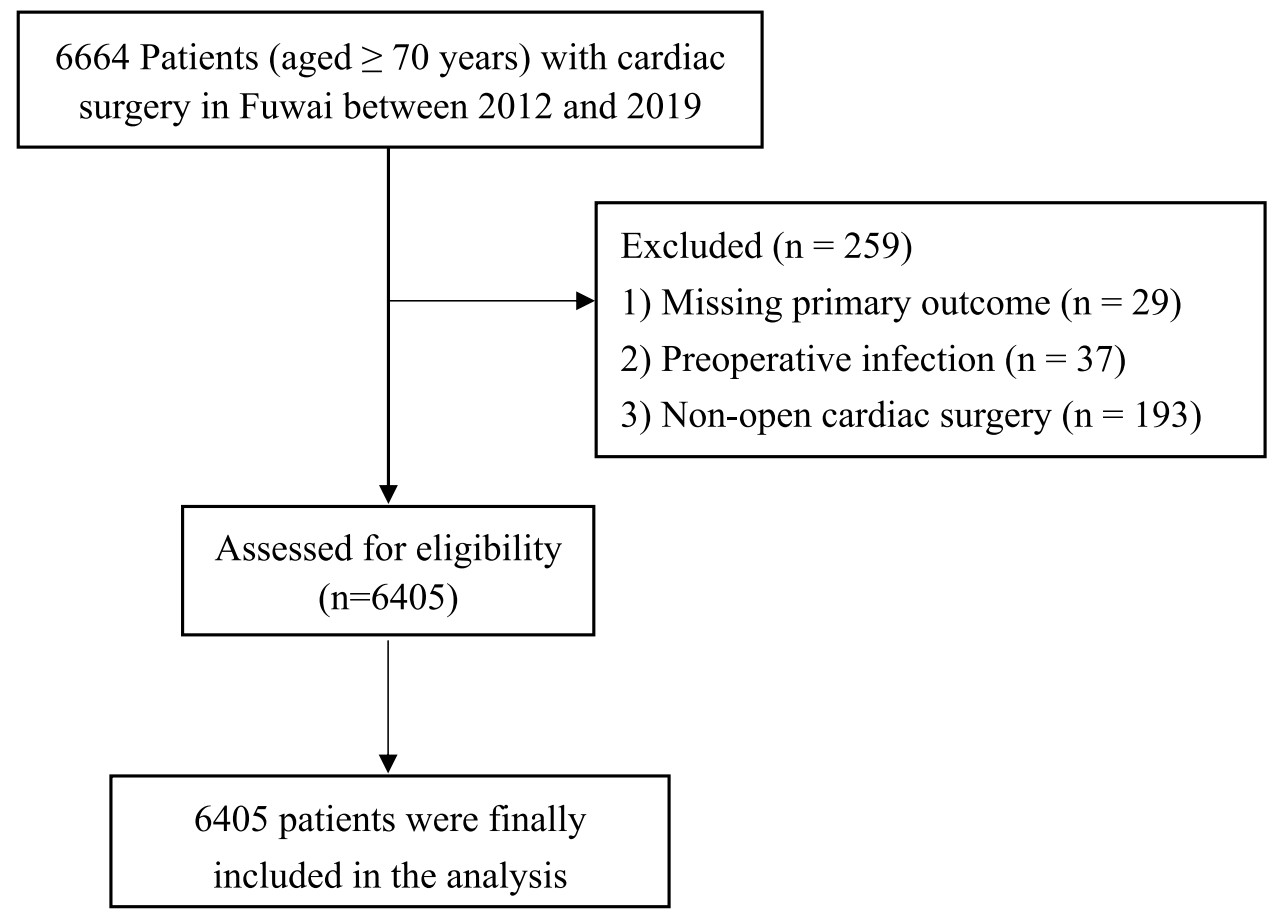

Figure I Flow chart of the study cohort. 
Microbiology cultures were routinely carried out during the perioperative period if clinical infection was suspected. Patients were admitted to the cardiac intensive care unit (ICU) with tracheal intubation following cardiac surgery.

\section{Study Outcomes}

The primary outcome was defined as hospital-acquired infection during the postoperative episodes until discharge, including pneumonia, urinary tract infections (UTIs) and surgical site infection (SSI). Infections were documented in a timely manner in medical records given attending clinicians suspected infectious potential based concomitantly on patient symptoms, biomarker examinations and microorganism culture. The length of ICU and total hospitalization stays were considered as secondary end points.

\section{Statistical Analysis}

Means with standard deviations and medians with interquartile ranges were calculated for all continuous outcomes, while frequency counts and percentages were calculated for categorical outcomes. Continuous outcomes were analyzed by Mann-Whitney $U$-tests, while categorical variables were analyzed by $\chi^{2}$ or Fisher's exact test as appropriate. Variables with a $P$ value less than 0.1 in the univariate logistic regression analysis were included in the final multivariate analysis. Based on the final results of multivariate logistic regression, a nomogram was built using the rms package in R software.

The discriminative performance of the nomogram was quantitatively assessed by the C-index, which was also a surrogate of the area under the receiver operating characteristic (AUROC) curve. The Hosmer-Lemeshow goodnessof-fit test was used to assess model calibration, and its nonsignificant statistical value presented good calibration. We further delineated calibration plots to graphically evaluate how close the nomogram predicted risk was to the actual risk. The model was internally validated for discrimination and calibration using 1000 bootstrap resamplings. ${ }^{11}$ Additionally, decision curve analysis (DCA) was performed in our study to determine the clinical usefulness of the predictive nomogram through the rmda package. With regard to clinical utility, DCA can facilitate quantifying the net benefits of predictive models in patients with HAI. ${ }^{12}$ All analyses were performed with the statistical software R for Mac 3.6.3 and SPSS for Mac 26.0.

\section{Results}

\section{Patients Characteristics}

Among 6405 patients recruited into the final analysis, the mean age was 73.6 years old, and $66.76 \%$ were male. The most common comorbidity was hypertension, occurring in 4128 patients (64.45\%). Table 1 shows the baseline characteristics of patients who had HAI and those who did not. Patients with postoperative HAI had longer hospital stays (25 days vs 18 days) and ICU stays (8 days vs 4 days).

\section{Incidence and Risk Factors for Hospital-Acquired Infection}

The incidence of postoperative HAI was 3.92\% (251/6405). Of the patients with HAIs, 229 patients had pneumonia, 14 patients had SSI, and 9 patients had UTI. In multivariable analysis, risk factors that were significantly associated with postoperative HAIs risk were smoking $(\mathrm{OR}=1.439,95 \% \mathrm{CI} 1.105-1.875)$, a history of myocardial infarction $(\mathrm{OR}=1.837$, 95\% CI 1.326-2.546), CPB use ( $\mathrm{OR}=1.487,95 \%$ CI 1.108-1.995), intraoperative erythrocytes transfusion ( $\mathrm{OR}=1.540$, 95\% CI 1.172-2.022), increasing days of preoperative hospitalization ( $\mathrm{OR}=1.186,95 \%$ CI 1.044-1.346), and prolonged duration of postoperative mechanical ventilation $(\mathrm{OR}=1.177,95 \%$ CI 1.139-1.215) (Table 2).

\section{Model Visualization and Performance}

A predictive nomogram for HAI after cardiac surgery was constructed following incorporating the 6 risk factors from multivariate logistic regression (Figure 2).

These 6 independent predictors separately with a point ranging from 0 to 100 were summed to generate the final total score, which was then converted into an individual probability of HAI. 
Table I Patients Characteristics

\begin{tabular}{|c|c|c|c|c|}
\hline Variable & $\begin{array}{c}\text { Total } \\
(n=6405)\end{array}$ & $\begin{array}{c}\text { No HAI } \\
(n=6154)\end{array}$ & $\begin{array}{c}\text { HAI } \\
(n=25 I)\end{array}$ & $P$ value \\
\hline \multicolumn{5}{|l|}{ Demographics } \\
\hline Male & $4276(66.76)$ & $4101(66.64)$ & 175 (69.72) & 0.339 \\
\hline Age (years) & $73.60(2.81)$ & $73.59(2.82)$ & $73.75(2.69)$ & 0.374 \\
\hline BMI $\left(\mathrm{kg} / \mathrm{m}^{2}\right)$ & $24.78(3.12)$ & $24.79(3.13)$ & $24.42(2.92)$ & 0.064 \\
\hline \multicolumn{5}{|l|}{ Comorbidities } \\
\hline Smoking (yes) & $2756(43.03)$ & $2628(42.70)$ & $128(51.00)$ & 0.011 \\
\hline Hypertension (yes) & $4128(64.45)$ & $3964(64.4 I)$ & $164(65.34)$ & 0.788 \\
\hline Diabetes (yes) & $1858(29.01)$ & $\mid 773(28.8 \mid)$ & $85(33.86)$ & 0.089 \\
\hline Hyperlipidemia (yes) & $3461(54.04)$ & 3329 (54.09) & $132(52.59)$ & 0.651 \\
\hline MI (yes) & $882(13.77)$ & $828(13.45)$ & $54(21.5 \mathrm{I})$ & 0.001 \\
\hline Atrial fibrillation (yes) & $699(10.91)$ & $660(10.72)$ & $39(15.54)$ & 0.022 \\
\hline PVD (yes) & $858(13.40)$ & $814(13.23)$ & $44(17.53)$ & 0.058 \\
\hline Stroke (yes) & $1905(29.74)$ & $1819(29.56)$ & $86(34.26)$ & 0.121 \\
\hline COPD (yes) & $377(5.89)$ & $363(5.90)$ & $14(5.58)$ & 0.832 \\
\hline Anemia (yes) & $2435(38.02)$ & $2331(37.88)$ & $104(41.43)$ & 0.260 \\
\hline Previous cardiac surgery (yes) & $197(3.08)$ & $182(2.96)$ & $15(5.98)$ & 0.013 \\
\hline \multicolumn{5}{|l|}{ Procedure details } \\
\hline CPB (yes) & $3744(58.45)$ & $3567(57.96)$ & 177 (70.52) & $<0.001$ \\
\hline CPB time (minutes) & $101[81-128]$ & $10 \mid[8 \mid-127]$ & $110[86-144]$ & 0.002 \\
\hline ACCT (minutes) & $7 \mid[54-92]$ & $70[53-91]$ & $78[55-104]$ & 0.006 \\
\hline Erythrocytes transfusion (yes) & $2145(33.49)$ & $2028(32.95)$ & $117(46.61)$ & $<0.001$ \\
\hline FFP transfusion (yes) & $858(13.40)$ & $812(13.19)$ & $46(18.33)$ & 0.023 \\
\hline Platelet transfusion (yes) & $309(4.82)$ & $282(4.58)$ & $27(10.76)$ & $<0.001$ \\
\hline Surgery type & & & & 0.021 \\
\hline Isolated CABG & $4527(70.68)$ & $4368(70.98)$ & 159 (63.35) & \\
\hline Isolated valve & 877 (13.69) & $834(13.55)$ & $43(17.13)$ & \\
\hline Aortic & $228(3.58)$ & $221(3.59)$ & $7(2.79)$ & \\
\hline Combined & $746($ (II.65) & 704 (II.44) & $42(16.73)$ & \\
\hline Others & $27(0.42)$ & $27(0.44)$ & $0(0)$ & \\
\hline \multicolumn{5}{|l|}{ Others } \\
\hline LVEF (\%) & $60.46(7.92)$ & $60.52(7.90)$ & $58.83(8.1 \mathrm{I})$ & 0.001 \\
\hline NYHA & & & & 0.429 \\
\hline 1 & $578(9.02)$ & $557(9.05)$ & $21(8.37)$ & \\
\hline ॥ & $3461(54.04)$ & $3327(54.06)$ & 134 (53.39) & \\
\hline III & $2191(34.21)$ & $2106(34.22)$ & $85(33.86)$ & \\
\hline IV & $175(2.73)$ & $164(2.66)$ & II (4.38) & \\
\hline Pre-operative hospitalization (days) & $7[5-11]$ & $7[5-11]$ & $7[5-13]$ & 0.237 \\
\hline \multicolumn{5}{|l|}{ Postoperative outcome } \\
\hline MV time (hours) & $19[16-27]$ & $19[16-26]$ & 34 [|9-9|] & $<0.001$ \\
\hline ICU days & $3[2-5]$ & $3[2-5]$ & $6[4-8]$ & $<0.001$ \\
\hline Total hospitalization (days) & $16[|3-2|]$ & $16[|3-2|]$ & $21[15-30]$ & $<0.001$ \\
\hline
\end{tabular}

Abbreviations: HAI, healthcare-associated infection; BMI, body mass index; MI, myocardial infarction; PVD, peripheral vascular disease; COPD; chronic pulmonary disease; LVEF, left ventricular ejection fraction; NYHA, New York Heart Association; CPB, cardiopulmonary bypass; ACCT, aortic cross clamp time; FFP fresh frozen plasma; CABG, coronary artery bypass graft surgery; MV, mechanical ventilation; ICU, intensive care unit.

As shown in Figure 3A, the AUROC of the predictive nomogram was 0.706 (95\% CI, 0.671 to 0.740 ). Internal validation using a bootstrapping technique with 1000 resamples presented an analogous result on discrimination, as the Harrell C-Index was 0.689. The derivation model presented good calibration, as the P value for the Hosmer-Lemeshow test was 0.139 . Additionally, internal validation also demonstrated a similar good calibration as the derivation model, which is delineated in Figure 3B. 
Table 2 Univariate and Multivariate Logistic Regression Analysis of Risk Factors for Healthcare-Associated Infection

\begin{tabular}{|c|c|c|c|c|}
\hline \multirow[t]{2}{*}{ Variable } & \multicolumn{2}{|l|}{ Univariate Analysis } & \multicolumn{2}{|l|}{ Multivariate Analysis } \\
\hline & Odds Ratio $(95 \% \mathrm{CI})$ & $P$ value & Odds Ratio* (95\% Cl) & $P$ value \\
\hline \multicolumn{5}{|l|}{ Demographics } \\
\hline Sex(male) & $1.153(0.876-1.157)$ & 0.310 & & \\
\hline Age & $1.020(0.976-1.065)$ & 0.374 & & \\
\hline BMI & $0.962(0.923-1.002)$ & 0.064 & & \\
\hline \multicolumn{5}{|l|}{ Comorbidities } \\
\hline Smoking (yes vs no) & $1.396(1.085-1.797)$ & 0.010 & $1.594(1.222-2.080)$ & 0.001 \\
\hline Hypertension (yes vs no) & 1.041 (0.799-I.358) & 0.764 & & \\
\hline Diabetes (yes vs no) & $1.265(0.969-1.653)$ & 0.084 & & \\
\hline Hyperlipidemia (yes vs no) & $0.94 \mid(0.731-1.212)$ & 0.639 & & \\
\hline MI (yes vs no) & $1.763(1.293-2.404)$ & $<0.001$ & $1.797(1.279-2.526)$ & $<0.001$ \\
\hline Atrial fibrillation (yes vs no) & $1.531(1.078-2.175)$ & 0.017 & & \\
\hline PVD (yes vs no) & $1.394(0.999-1.947)$ & 0.051 & & \\
\hline Stroke (yes vs no) & $1.242(0.952-1.62 I)$ & 0.111 & & \\
\hline COPD (yes vs no) & $0.942(0.544-1.632)$ & 0.832 & & \\
\hline Anemia (yes vs no) & $1.160(0.898-1.499)$ & 0.256 & & \\
\hline Previous cardiac surgery (yes vs no) & $2.086(1.213-3.587)$ & 0.008 & & \\
\hline \multicolumn{5}{|l|}{ Others } \\
\hline LVEF & $0.975(0.96 \mathrm{I}-0.990)$ & 0.001 & & \\
\hline NYHA (III, IV) & $1.060(0.817-1.374)$ & 0.662 & & \\
\hline Pre-operative hospitalization (per week) & $1.207(1.069-1.364)$ & 0.002 & $1.186(1.044-1.346)$ & 0.009 \\
\hline \multicolumn{5}{|l|}{ Procedure details } \\
\hline АCCT (per $30 \mathrm{~min}$ ) & $1.215(1.130-1.307)$ & $<0.001$ & & \\
\hline CPB (yes vs no) & $1.735(1.316-2.286)$ & $<0.001$ & I.487 (I.108-I.995) & 0.008 \\
\hline Erythrocytes transfusion (yes vs no) & $1.776(1.378-2.289)$ & $<0.001$ & $1.540(1.172-2.022)$ & 0.002 \\
\hline FFP transfusion (yes vs no) & $1.476(1.063-2.050)$ & 0.020 & & \\
\hline Platelet transfusion (yes vs no) & $2.510(1.654-3.808)$ & $<0.001$ & & \\
\hline Surgery type & $1.146(1.026-1.280)$ & 0.016 & & \\
\hline \multicolumn{5}{|l|}{ Postoperative outcome } \\
\hline MV time (per 24 hours) & $1.195(1.157-1.233)$ & $<0.001$ & $1.177(1.139-1.215)$ & $<0.001$ \\
\hline
\end{tabular}

Notes: *Adjusted for previous cardiac surgery, diabetes, BMI, PVD, Atrial fibrillation, LVEF, CPB time, ACCT, FFP transfusion, surgery type and platelet transfusion.

Abbreviations: BMI, body mass index; MI, myocardial infarction; PVD, peripheral vascular disease; COPD; chronic pulmonary disease; LVEF, left ventricular ejection fraction; NYHA, New York Heart Association; CPB, cardiopulmonary bypass; ACCT, aortic cross clamp time; FFP, fresh frozen plasma; CABG, coronary artery bypass graft surgery; MV, mechanical ventilation; ICU, intensive care unit.

\section{Clinical Application}

The decision curves of the nomogram revealed good clinical utility both in the derivation cohort and the validation cohort with bootstrapping (Figure 4A and B). Using the nomogram to predict HAI with the threshold probability $>0.5 \%$ added more benefit than either the extreme situation of diagnosing HAI in all patients or none.

\section{Discussion}

The HAI rate was $3.9 \%$ in our study, which was slightly lower than the findings of other studies focusing on cardiac surgery. ${ }^{13,14}$ During the perioperative period, risk factors that could be modified aimed at ameliorating postoperative HAI were smoking, erythrocytes transfusion, length of preoperative hospital stay and duration of postoperative mechanical ventilation. CPB use and myocardial infarction history were an additional 2 uncontrolled contributors. The nomogram generated using 6 risk contributors with optimal discrimination and calibration should be considered a feasible and convenient tool in clinical scenarios to predict postoperative HAI and then achieve early intervention among elderly individuals. 


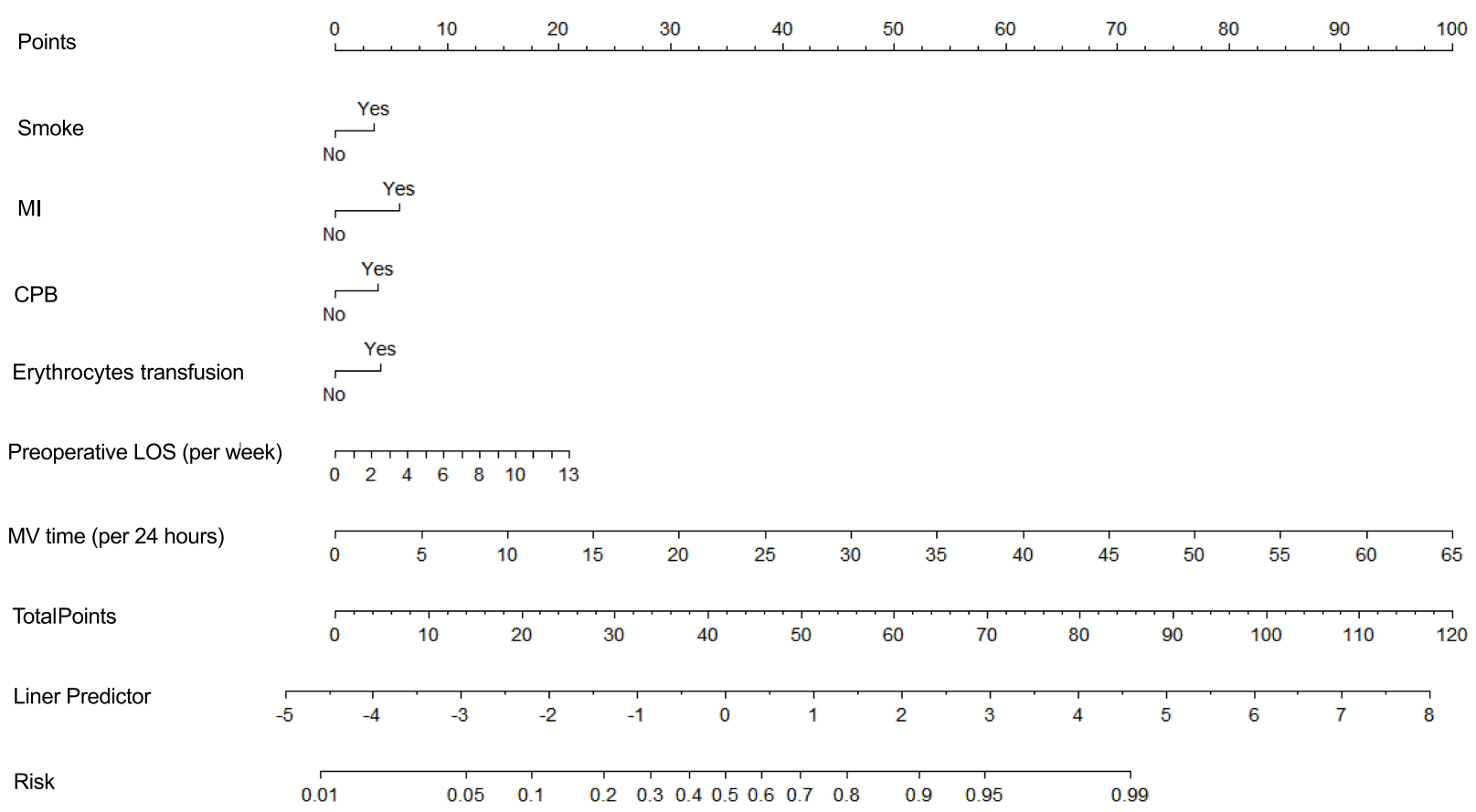

Figure 2 Nomogram predicting the risk of HAls based on independent risk factors identified from multivariate logistic regression analysis.

Abbreviations: HAI, hospital-acquired infection; MI, myocardial infarction; CPB, cardiopulmonary bypass; LOS, length of stay; MV, mechanical ventilation.

A

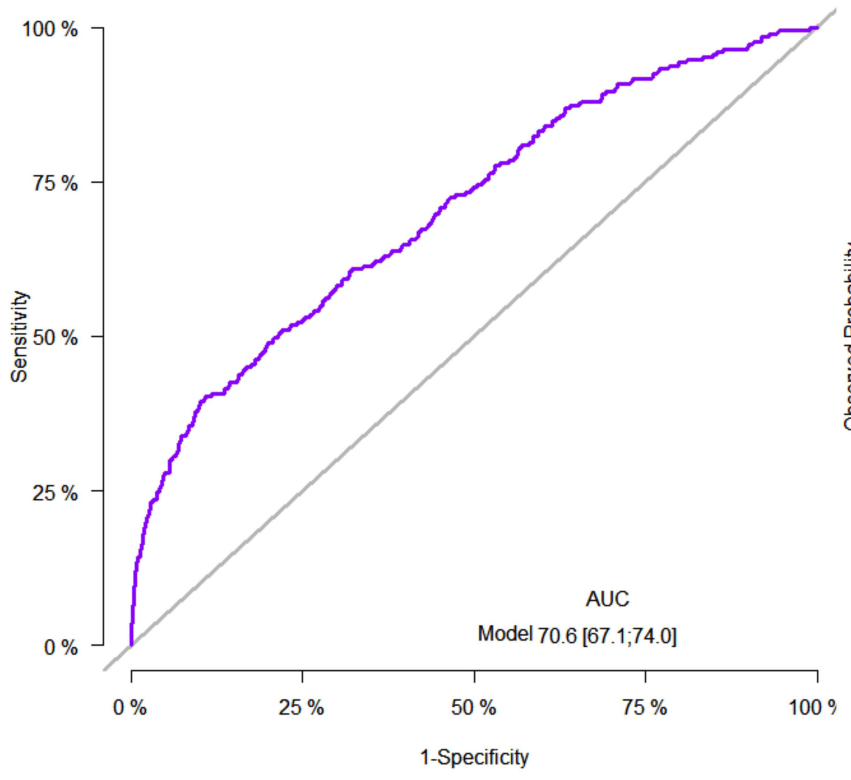

B

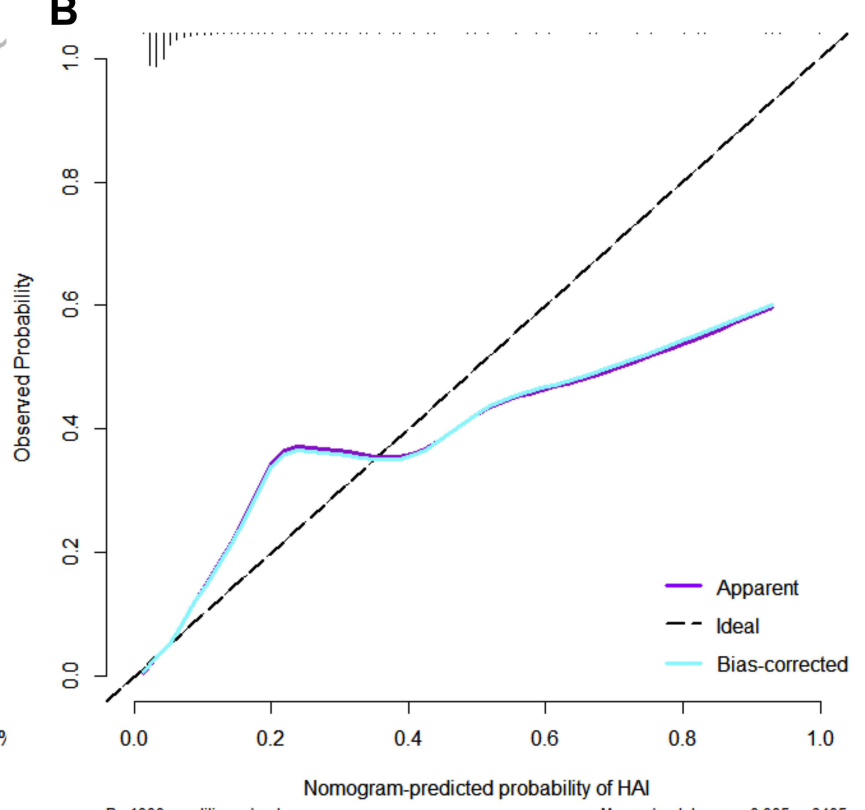

$B=1000$ repetitions, boot

Figure 3 The receiver operating characteristic curve and calibration curve. (A). ROC curve of the nomogram. (B). Calibration curve of derivation cohorts and internal bootstrap validation cohorts. In (B), X-axis is the nomogram-predicted probability; Y-axis is the actually observed probability. The dashed black line indicates a perfect prediction by an ideal model. The purple solid line represents the calibration of established nomogram, while blue solid line is bias-corrected with bootstrapping technique. Abbreviation: ROC, receiver operating characteristic. 

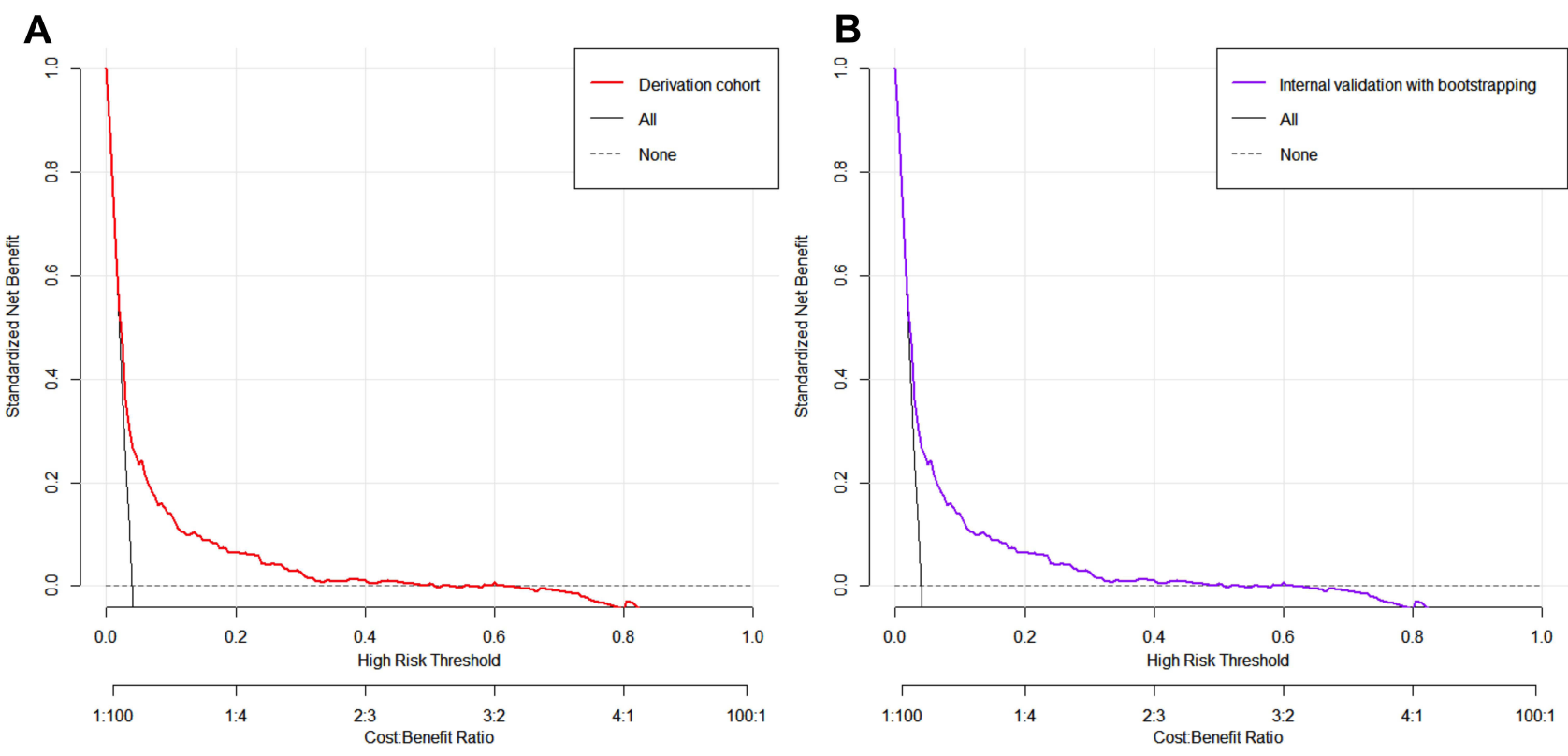

Figure 4 Decision curve analyses for the HAls nomogram. (A). The derivation cohort. (B). The internal bootstrap validation. Black solid line indicates net benefit of all patients developing HAls postoperatively. Gray dotted line indicates net benefit of no patients developing HAls postoperatively. Red and purple solid lines indicate net benefit of the nomogram derived from derivation cohorts and internal bootstrap validation cohorts. Abbreviation: HAI, hospital-acquired infection.

Individuals with older age are inclined to have a dysregulated immune system that responds fiercely to infectious pathogens. ${ }^{15}$ Early identification and effective treatments for postoperative HAI have repeatedly been shown to decrease associated mortality. ${ }^{16,17}$ However, controlling risk factors before HAI exposure is more promising than focusing on medical interventions once infection has occurred. Smokers had a higher likelihood of encountering postoperative infectious complications than nonsmokers, partly due to their impaired immune function. ${ }^{18,19}$ Smoking cessation before surgery, considered conventionally a major potential target to improve clinical outcomes, is highly recommended in the guidelines for perioperative care in the cardiac population by the Enhanced Recovery After Surgery Society. ${ }^{20}$

Several studies have demonstrated that HAI was significantly associated with red blood cell (RBC) transfusion, ${ }^{8,14,21,22}$ consistent with the findings of the present study. Likosky et al used the Michigan Society of Thoracic and Cardiovascular Surgeons Quality Collaborative database to assess the relationship between RBC transfusion and postoperative pneumonia after CABG. The authors found that patients receiving only 2 units of RBCs carried a 2-fold greater risk for pneumonia development. ${ }^{23}$ Developing and subsequently adhering to blood conservation protocols, composed of cell salvage, minimizing hemodilution and preoperative hemoglobin optimization, are of great clinical benefits. They were dedicated not only to reducing blood transfusion requirements but also to improving patient clinical outcomes. ${ }^{24}$

We found that the risk for infection associated with episodes of mechanical ventilation was dose-dependent, with an $18 \%$ increase in infection per 24-hour increment in the ventilation time. Additionally, pneumonia was the primary component of HAI, with an incidence of $91.2 \%$, which may be primarily attributed to prolonged mechanical ventilation. Ailawadi revealed that compared with patients who were extubated within 24 hours, those intubated for 24 to 48 hours or longer had a 2.83 -fold and 4.67-fold increased risk of pneumonia, respectively. ${ }^{25}$ Our study therefore reinforces the importance of early extubation after cardiac surgery, a crucial approach towards realizing postoperative enhanced recovery and enabling early mobilization and oral nutrition. Short-acting and low-dose narcotics may facilitate early endotracheal extubation, and a time-directed extubation protocol can improve the efficiency of weaning from mechanical ventilation as well. ${ }^{26}$

An increase in postoperative infection in parallel with extended preoperative hospitalized days has been confirmed. ${ }^{27-29}$ Hospitalization may predispose patients to skin and respiratory colonization with more virulent hospital-based pathogens. ${ }^{30}$ 
Patients with a longer preoperative length of stay are more prone to mediastinitis after cardiac surgery. ${ }^{31}$ Of the patients undergoing coronary artery bypass graft, only 53\% underwent surgery on the day of hospital admission. The overall infection risk after coronary artery bypass graft increased 3.2-fold when the procedure was delayed by 6 to 10 days. ${ }^{32} \mathrm{~A}$ perweek increase in hospital stay preoperatively was associated with a $19 \%$ increased risk of HAI in our study. However, the number of days hospitalized prior to surgery is a potentially adjustable factor in clinical practice. Thus, minimizing preoperative hospitalization is a prudent precaution that may help to reduce the likelihood of developing HAI. In contrast, reducing the length of hospitalization prior to surgery for elderly patients remains a challenge for clinicians, as they require dedicated care given the high frequency of comorbidities.

To the best of our knowledge, this is the first study to establish a nomogram of postoperative HAI for elderly individuals undergoing cardiac surgery. This nomogram would allow the individual risk probability of HAI to be scored conveniently in daily clinical practice after incorporating 4 modifiable and 2 unmodifiable clinical factors. In addition, the nomogram could help cardiologists to differentiate the risk management of HAI patients by weighing net benefits, offering clinicians the ability to make more individualized treatment decisions.

This study has several limitations. First, it has a retrospective design, which has inherent difficulties in diagnosing infections. Only documented cases of HAI were taken into account, which could lead to an underestimation of the real rate of HAI. Second, we could not determine whether subtypes of HAI may vary based on different sets of preoperative risk factors. Third, whether our results can be generalized to other centers is known due to disparities in antibiotic usage. The present study does not provide any information on microorganism distribution, which could be used for the analysis of HAI etiology. Additional prospective studies focused on comparing the predicted and real rates of postoperative HAI using this nomogram are needed.

\section{Conclusions}

In summary, the results of our study revealed that modifiable risk factors such as smoking, erythrocytes transfusion, preoperative length of hospital stay and postoperative mechanical ventilation and unmodifiable risk factors such as CPB use and a history of myocardial ischemia can cause postoperative HAI in elderly patients. A simple risk prediction model incorporating these factors can help guide clinical decision making, patient counseling and treatment planning.

\section{Abbreviations}

HAI, Hospital-acquired infection; DCA, decision curve analysis; UTIs, urinary tract infections; SSI, surgical site infection; AUROC, area under the receiver operating characteristic curve; ICU, cardiac intensive care unit; CPB, cardiopulmonary bypass; RBC, red blood cell.

\section{Data Sharing Statement}

The dataset generated and analyzed during the current study is available from the corresponding author upon reasonable request.

\section{Ethics Approval and Consent to Participate}

This retrospective study was approved by the Ethics Committee of Fuwai hospital with waived written consent, and was conducted according to the World Medical Association Declaration of Helsinki. Because all included data were anonymous and retrospective, the requirement that patients give informed consent was waived.

\section{Acknowledgments}

We thank all participants for their contribution to this study.

\section{Author Contributions}

All authors met the following conditions: 
1. Made a significant contribution to the work reported, whether that is in the conception, study design, execution, acquisition of data, analysis and interpretation, or in all these areas.

2. Have drafted or written, or substantially revised or critically reviewed the article.

3. Have agreed on the journal to which the article will be submitted.

4. Reviewed and agreed on all versions of the article before submission, during revision, the final version accepted for publication, and any significant changes introduced at the proofing stage.

5. Agree to take responsibility and be accountable for the contents of the article.

\section{Funding}

This work is supported by the Chinese Academy of Medical Sciences Central Public Welfare Scientific Research Institute Basal Research Expenses (2019XK320052) and Special Fund for the Development of Characteristic Disciplines in Fuwai Hospital (2019-F05A).

\section{Disclosure}

The authors report no conflicts of interest in this work.

\section{References}

1. Speziale G, Nasso G, Barattoni MC, et al. Short-term and long-term results of cardiac surgery in elderly and very elderly patients. $J$ Thorac Cardiovasc Surg. 2011;141(3):725-731, 731 e721. doi:10.1016/j.jtcvs.2010.05.010

2. Afilalo J, Steele R, Manning WJ, et al. Derivation and validation of prognosis-based age cutoffs to define elderly in cardiac surgery. Circ Cardiovasc Qual Outcomes. 2016;9(4):424-431. doi:10.1161/CIRCOUTCOMES.115.002409

3. Afilalo J, Mottillo S, Eisenberg MJ, et al. Addition of frailty and disability to cardiac surgery risk scores identifies elderly patients at high risk of mortality or major morbidity. Circ Cardiovasc Qual Outcomes. 2012;5(2):222-228. doi:10.1161/CIRCOUTCOMES.111.963157

4. Greco G, Shi W, Michler R, et al. Costs associated with health care-associated infections in cardiac surgery. J Am Coll Cardiol. 2015;65(1):15-23. doi:10.1016/j.jacc.2014.09.079

5. Massart N, Mansour A, Ross J, et al. Mortality due to hospital-acquired infection after cardiac surgery. J Thorac Cardiovasc Surg. 2020. doi:10.1016/j.jtcvs.2020.08.094

6. de la Varga-martínez O, Gómez-Sánchez E, Muñoz M, et al. Impact of nosocomial infections on patient mortality following cardiac surgery. J Clin Anesth. 2020;69:110104. doi:10.1016/j.jclinane.2020.110104

7. Kollef M, Torres A, Shorr A, et al. Nosocomial Infection. Crit Care Med. 2021;49(2):169-187. doi:10.1097/CCM.0000000000004783

8. Mazzeffi M, Gammie J, Taylor B, et al. Healthcare-associated infections in cardiac surgery patients with prolonged intensive care unit stay. Ann Thorac Surg. 2017;103(4):1165-1170. doi:10.1016/j.athoracsur.2016.12.041

9. Shariat S, Capitanio U, Jeldres C, et al. Can nomograms be superior to other prediction tools? BJU Int. 2009;103(4):492-495; discussion 495-497. doi:10.1111/j.1464-410X.2008.08073.x

10. Balachandran V, Gonen M, Smith J, et al. Nomograms in oncology: more than meets the eye. Lancet Oncol. 2015;16(4):e173-180. doi:10.1016/ S1470-2045(14)71116-7

11. Harrell F, Lee K, Mark D. Multivariable prognostic models: issues in developing models, evaluating assumptions and adequacy, and measuring and reducing errors. Stat Med. 1996;15(4):361-387. doi:10.1002/(SICI)1097-0258(19960229)15:4<361::AID-SIM168>3.0.CO;2-4

12. Van Calster B, Wynants L, Verbeek J, et al. Reporting and interpreting decision curve analysis: a guide for investigators. Eur Urol. 2018;74 (6):796-804. doi:10.1016/j.eururo.2018.08.038

13. Gelijns A, Moskowitz A, Acker M, et al. Management practices and major infections after cardiac surgery. J Am Coll Cardiol. 2014;64(4):372-381. doi:10.1016/j.jacc.2014.04.052

14. Horvath K, Acker M, Chang H, et al. Blood transfusion and infection after cardiac surgery. Ann Thorac Surg. 2013;95(6):2194-2201. doi:10.1016/j. athoracsur.2012.11.078

15. Castle SC. Clinical relevance of age-related immune dysfunction. Clin Infect Dis. 2000;31(2):578-585. doi:10.1086/313947

16. Kollef M, Sharpless L, Vlasnik J, et al. The impact of nosocomial infections on patient outcomes following cardiac surgery. Chest. 1997;112 (3):666-675. doi:10.1378/chest.112.3.666

17. Lola I, Levidiotou S, Petrou A, et al. Are there independent predisposing factors for postoperative infections following open heart surgery? J Cardiothorac Surg. 2011;6:151. doi:10.1186/1749-8090-6-151

18. Sørensen L. Wound healing and infection in surgery. The clinical impact of smoking and smoking cessation: a systematic review and meta-analysis. Arch Surg. 2012;147(4):373-383. doi:10.1001/archsurg.2012.5

19. Tønnesen H, Nielsen P, Lauritzen J, et al. Smoking and alcohol intervention before surgery: evidence for best practice. Br J Anaesth. 2009;102 (3):297-306. doi:10.1093/bja/aen401

20. Engelman D, Ben Ali W, Williams J, et al. Guidelines for perioperative care in cardiac surgery: enhanced recovery after surgery society recommendations. JAMA Surg. 2019;154(8):755-766. doi:10.1001/jamasurg.2019.1153

21. Banbury M, Brizzio M, Rajeswaran J, et al. Transfusion increases the risk of postoperative infection after cardiovascular surgery. $J$ Am Coll Surg. 2006;202(1):131-138. doi:10.1016/j.jamcollsurg.2005.08.028

22. Ming Y, Liu J, Zhang F, et al. Transfusion of red blood cells, fresh frozen plasma, or platelets is associated with mortality and infection after cardiac surgery in a dose-dependent manner. Anesth Analg. 2020;130(2):488-497. doi:10.1213/ANE.0000000000004528 
23. Likosky D, Paone G, Zhang M, et al. Red blood cell transfusions impact pneumonia rates after coronary artery bypass grafting. Ann Thorac Surg. 2015;100(3):794-800; discussion 801. doi:10.1016/j.athoracsur.2015.03.089

24. Ad N, Holmes S, Patel J, et al. The impact of a multidisciplinary blood conservation protocol on patient outcomes and cost after cardiac surgery. $J$ Thorac Cardiovasc Surg. 2017;153(3):597-605.e591. doi:10.1016/j.jtcvs.2016.10.083

25. Ailawadi G, Chang H, O'Gara P, et al. Pneumonia after cardiac surgery: experience of the National Institutes of Health/Canadian Institutes of Health Research Cardiothoracic Surgical Trials Network. J Thorac Cardiovasc Surg. 2017;153(6):1384-1391.e1383. doi:10.1016/j. jtcvs.2016.12.055

26. McCarthy C, Fletcher N. Early extubation in enhanced recovery from cardiac surgery. Crit Care Clin. 2020;36(4):663-674. doi:10.1016/j. ccc.2020.06.005

27. Lee Y, Feng M, Wu L, et al. Outcome and risk factors associated with surgical site infections after cardiac surgery in a Taiwan medical center. J Microbiol Immunol Infect. 2010;43(5):378-385. doi:10.1016/S1684-1182(10)60060-6

28. Bueno Cavanillas A, Rodrìguez-Contreras R, Delgado Rodriguez M, et al. Preoperative stay as a risk factor for nosocomial infection. Eur J Epidemiol. 1991;7(6):670-676. doi:10.1007/BF00218680

29. Hessels A, Agarwal M, Liu J, et al. Incidence and risk factors for health-care associated infections after hip operation. Surg Infect (Larchmt). 2016;17(6):761-765. doi:10.1089/sur.2016.062

30. Sulzgruber P, Schnaubelt S, Koller L, et al. An extended duration of the pre-operative hospitalization is associated with an increased risk of healthcare-associated infections after cardiac surgery. Sci Rep. 2020;10(1):8006. doi:10.1038/s41598-020-65019-8

31. Leung Wai Sang S, Chaturvedi R, Alam A, et al. Preoperative hospital length of stay as a modifiable risk factor for mediastinitis after cardiac surgery. J Cardiothorac Surg. 2013;8:45. doi:10.1186/1749-8090-8-45

32. Vogel T, Dombrovskiy V, Lowry S. In-hospital delay of elective surgery for high volume procedures: the impact on infectious complications. $J$ Am Coll Surg. 2010;211(6):784-790. doi:10.1016/j.jamcollsurg.2010.08.009

\section{Publish your work in this journal}

Clinical Interventions in Aging is an international, peer-reviewed journal focusing on evidence-based reports on the value or lack thereof of treatments intended to prevent or delay the onset of maladaptive correlates of aging in human beings. This journal is indexed on PubMed Central, MedLine, CAS, Scopus and the Elsevier Bibliographic databases. The manuscript management system is completely online and includes a very quick and fair peer-review system, which is all easy to use. Visit http://www.dovepress.com/testimonials.php to read real quotes from published authors.

Submit your manuscript here: https://www.dovepress.com/clinical-interventions-in-aging-journal 